\title{
Prophylactic antibiotics to prevent pneumonia and other complications after measles: community based randomised double blind placebo controlled trial in Guinea-Bissau
}

\author{
May-Lill Garly, Carlitos Balé, Cesário Lourenco Martins, Hilton C Whittle, Jens Nielsen, Ida M Lisse, Peter Aaby
}

\begin{abstract}
Objective To investigate whether prophylactic antibiotics can prevent complications of measles.

Design Community based, randomised, double blind, placebo controlled trial.

Setting Bandim Health Project study area in Bissau, Guinea-Bissau, west Africa.

Participants 84 patients with measles during a measles epidemic in Bissau in 1998 (fewer than originally planned owing to interruption by war).

Interventions Sulfamethoxazole-trimethoprim (co-trimoxazole) or placebo for seven days.

Main outcome measures Pneumonia and admission to hospital. Also weight change during the first month of infection, diarrhoea, severe fever, oral thrush, stomatitis, conjunctivitis, and otitis media.

Results The median age of the patients with measles was 5.4 (range 0.49-24.8) years. One of 46 participants who received co-trimoxazole developed pneumonia, in contrast to six of 38 participants who received placebo (odds ratio $0.08(95 \%$ confidence interval 0 to 0.56 ), adjusted for age group). The number needed to treat was 7 (4 to 48). All three participants admitted to hospital had received placebo $(\mathrm{P}=0.09)$. The weight gain during the first month after inclusion was 15 (2-29) $\mathrm{g} /$ day in the placebo group and 32 (23-42) $\mathrm{g}$ /day in the co-trimoxazole group $(\mathrm{P}=0.04$, adjusted for age group, weight for age at inclusion, measles vaccination status, and duration of disease). Significantly less conjunctivitis occurred among recipients of co-trimoxazole than placebo, as well as a non-significant tendency to less diarrhoea, severe fever, oral thrush, and stomatitis. Complications of otitis media were the same in the two groups.

Conclusions The group that received prophylactic antibiotics had less pneumonia and conjunctivitis and had significantly higher weight gains in the month after inclusion. The results indicate that prophylactic antibiotics may have an important role in the management of measles infection in low income countries.

Trial registration Clinical trials NCT001168532.
\end{abstract}

\section{Introduction}

Despite intense efforts to eradicate it, measles still infects 30-40 million people worldwide and causes half a million deaths a year. ${ }^{1}$ It is the leading killer among vaccine preventable diseases and causes an estimated $44 \%$ of the 1.7 million vaccine prevent- able deaths among children each year. ${ }^{2}$ The case fatality rate of measles in developing countries is high, particularly among infants, and reaches $30 \%$ among patients admitted to hospital. ${ }^{3}$ Even in affluent countries, the complication rate is high and epidemics cause severe morbidity, permanent sequelae, and death. ${ }^{4}$ Unvaccinated people are at much higher risk of getting measles than are vaccinated people, but primary and secondary vaccine failures do occur, although vaccinated people tend to get milder measles and fewer complications. ${ }^{5}$

Before the era of measles vaccination, measles was often treated with prophylactic antibiotics at the primary healthcare level, even when complications had not yet developed. ${ }^{6}$ Trials of prophylactic antibiotics in measles infection were made several years ago, some of them randomised, but none of them complied with the current standards for design of a randomised controlled trial. ${ }^{7}$ In 1987 a project in Senegal implemented routine prophylactic antibiotics (co-trimoxazole for seven days) for all children under 3 years of age seen within the first two weeks after the onset of symptoms of measles. ${ }^{8}$ The study found a twofold reduction in the case fatality rate for measles in the cohort that received prophylactic antibiotics compared with historical controls. Furthermore, children aged under 3 years who had received prophylactic antibiotics were less likely to have respiratory symptoms on days 8-15 than were children of the same age group who had not received prophylactic antibiotics (relative risk $0.37,95 \%$ confidence interval 0.15 to 0.94 ). On this background, the World Health Organization proposed that a priority for measles research should be a randomised, double blind, placebo controlled trial of prophylactic antibiotics in measles. ${ }^{9}$ Here we report results from such a trial done in Guinea-Bissau in 1998. The trial was stopped prematurely because of the civil war in Guinea-Bissau in 1998-9. We used co-trimoxazole as the active drug, as it was WHO's recommended first line drug against pneumonia when the trial was planned.

\section{Methods}

\section{Setting}

The study took place in 1998 in Bissau, the capital of Guinea-Bissau, west Africa, under the auspices of the Bandim Health Project. ${ }^{10}$ The project's surveillance system registers pregnancies, births, deaths, infections, and vaccinations. Coverage of measles vaccine in the study area was $68 \%$ before the start of the two dose measles vaccination trials in 1995; after this it rose to $89 \% .^{11}$ 


\section{Measles surveillance}

We detected patients with measles and suspected measles through the surveillance system, weekly morbidity surveillance for the youngest children, consultations at the two health centres, and hospital admissions at the paediatric department in Bissau. We searched for additional cases in houses surrounding the homes of detected patients.

\section{Diagnosis of measles}

Project physicians diagnosed measles. Diagnosis of probable measles was based on the presence of at least one of the following symptoms: a typical maculo-papular rash; Koplik's spots, a typical desquamation found to be a reliable indicator of measles infection $^{12}$; and high fever $\left(>38^{\circ} \mathrm{C}\right) 5-15$ days after contact with a person known to have measles.

\section{Diagnosis of pneumonia}

Physicians referred patients with measles for chest radiography on suspicion of pneumonia by using the following screening signs: fast breathing defined as a respiratory rate $>60$ per minute, intercostal indrawings, diminished or absent air entry, bronchial breathing, crepitation, or dullness to percussion (fig 1). The tuberculosis hospital in Bissau did the $\mathrm{x}$ rays.

\section{Randomisation}

Ferraton produced co-trimoxazole and placebo, and Scanpharm A/S, Denmark delivered them. Ferraton produced a total of 400 containers of 42 paediatric tablets each and 200 containers of 28 tablets for adults. A person not involved in the study used dBASE IV to produce a list of 600 random numbers. Scanpharm used this list to mark the containers in accordance with the content.

We used the trial drug containers in numerical order, starting with 1 for paediatric tablets and 401 for adults. Participants up to 5 years of age or who weighed up to $18 \mathrm{~kg}$ received paediatric tablets, and participants aged 6 years and above or who weighed more than $18 \mathrm{~kg}$ received adults' tablets. We broke the randomisation code only after data analysis was complete.

\section{Procedures}

We started the study in January 1998. The inclusion criteria were a clinical diagnosis of probable measles in the prodromal phase or within the first seven days after the onset of rash. We excluded pregnant and nursing women, children less than 2 months old, patients who needed urgent referral to the hospital, patients with radiographs showing consolidated lung infection, and patients with other bacterial infections that needed systemic antibiotic treatment (fig 1). All patients who did not meet the inclusion criteria received standard care according to local recommendations.

We included patients if verbal consent was obtained. The duration of treatment was 7 days. All patients included received a printed consent form explaining the study. A physician visited participants twice a week during the first two weeks and once a

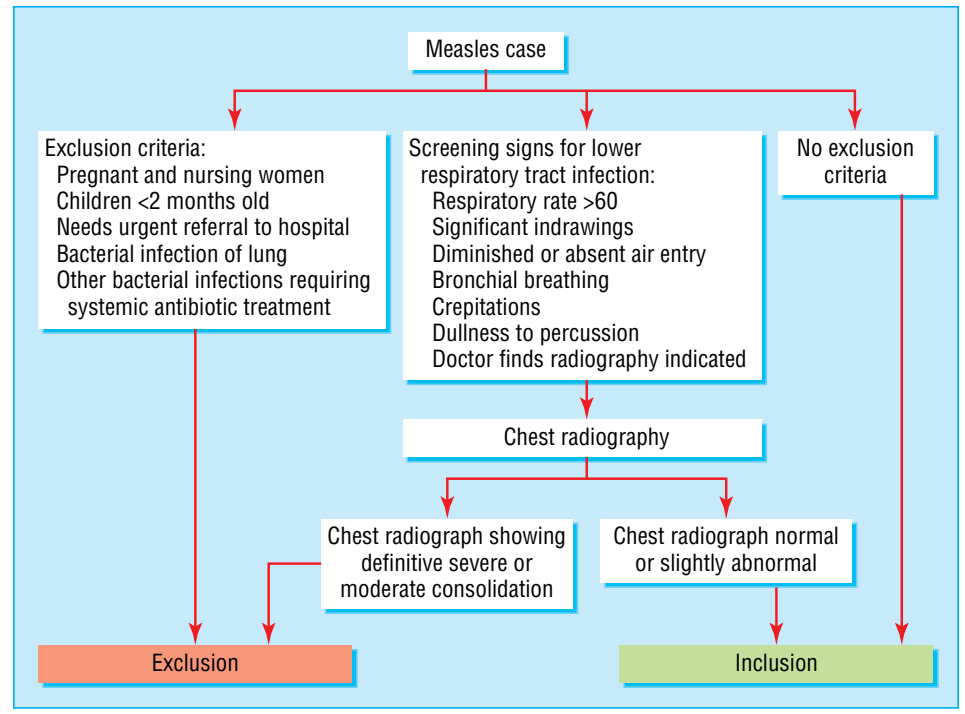

Fig 1 Flowchart of decision algorithm on inclusion or exclusion of patients with measles

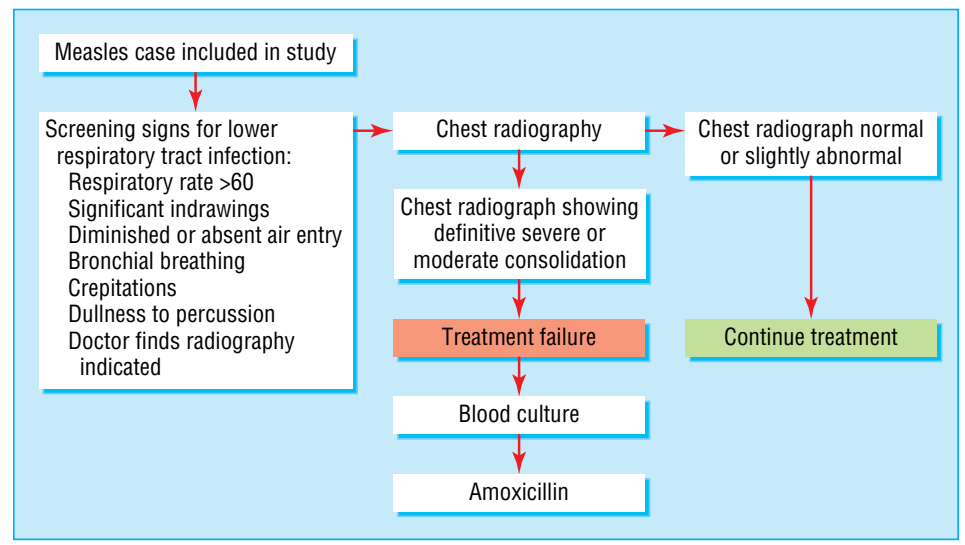

Fig 2 Flowchart of decision on case management and treatment failure 


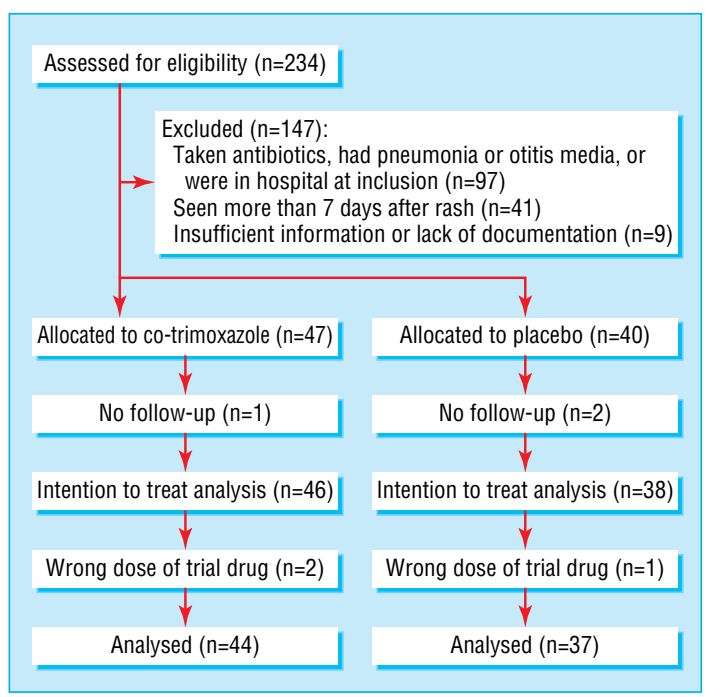

Fig 3 Consort flowchart

week during weeks three and four. If signs of bacterial pneumonia had developed, chest radiography was done. The result of chest radiography determined further case management (fig 2). If the physician suspected bacterial pneumonia, a blood culture was done, if possible, and antibiotic treatment was changed to amoxicillin. If Gram negative septicaemia or staphylococcal pneumonia was suspected, a blood culture was done and the patient was transferred to hospital and treated with parenteral ampicillin and gentamycin.

\section{Laboratory analyses}

Measles antibodies-We took two blood specimens four to six weeks apart. We stored serum at $-20^{\circ} \mathrm{C}$ until the MRC Laboratories in the Gambia did IgG measles antibody analysis, by measles haemagglutination inhibition test. ${ }^{13}$ If only one sample was available, or the IgG result was not conclusive, an IgM capture enzyme linked immunosorbant assay (ELISA) test was done. ${ }^{14}$ We considered a fourfold increase or decrease in IgG antibody concentration or a positive IgM test to be serological confirmation.

Blood culture-The National Laboratory in Bissau analysed blood cultures.

\section{Analytical methods}

Outcome measures-The main outcome measure was treatment failure due to pneumonia, admission to hospital, or both. Other outcome measures were weight change during the first month of infection, diarrhoea, severe fever, oral thrush, stomatitis, conjunctivitis, and otitis media. We coded the duration of disease in three groups: rash before enrolment, rash at enrolment or up to two days after, and rash on day 3-7 after enrolment. We divided age into five groups: 2-11 months, 1-2 years, 3-4 years, 5-17 years, and 18 years or over. We grouped participants into two groups according to vaccination status: vaccinated (had one or two vaccination dates or claimed previous measles vaccination) and unvaccinated. We divided weight for age $\mathrm{z}$ score at inclusion into less than -2 and -2 or above. We adjusted main analyses for age group, vaccination status, weight for age $\mathrm{z}$ score, and duration of disease (from onset of rash), as we expected the likelihood of an effect to be highest for participants treated early. We based the main analyses on intention to treat, including three participants who got the wrong dose of trial drug (fig 3). We defined a death from measles as a death occurring within 30 days after the rash.
Statistical analysis-We used SAS 9.1.3 to analyse data by logistic regression and non-parametric Wilcoxon two sample test. We used Fisher's exact test in sparse tables and profile likelihood to calculate confidence intervals. The number needed to treat is the inverse of the absolute risk reduction. We calculated absolute risk reduction by subtracting the pneumonia rate in the co-trimoxazole group from the pneumonia rate in placebo group. We used EPI-Info Nutrition to calculate weight for age $\mathrm{z}$ scores. We excluded three participants older than 18 years and six without weight data at the first visit from anthropometric analyses, which thus included 75 participants. We analysed weight change in a mixed linear model.

Power calculation-Assuming a frequency of lower respiratory infections or admission to hospital of at least 35\% in measles, using a significance level of $95 \%$ and a power of $80 \%$, a sample size of 218 should detect a $50 \%$ reduction in lung complications. We enrolled 84 participants between January and April 1998. A civil war broke out on 7 June 1998, and we had to close the study. We made an attempt to reopen the study in 2000 after the war, but this was not possible because of strikes by field workers.

\section{Results}

\section{8 measles epidemic}

Among 234 patients with measles evaluated for study entry, 84 entered the study and 150 did not. Reasons for exclusion were: $44 \%(\mathrm{n}=66)$ had taken antibiotics, $17 \%(25)$ had pneumonia, $27 \%$ (41) were more than seven days after rash or asymptomatic, $5 \%$ (7) had insufficient information, 2\% (3) had otitis media, 2\% (3) were in hospital with measles (and only examined later by the project), $2 \%$ (3) had only one visit (of whom two were visitors in the project area), and 1\% (2) lacked proper documentation (fig $3)$.

\section{Study population}

We included 84 patients with measles in the analyses. Median age at inclusion was 5.4 (range 0.49-24.8) years. Fifty five per cent received co-trimoxazole, and $45 \%$ received placebo (table 1). A higher proportion of participants in the co-trimoxazole group than in the placebo group were malnourished (weight for age $\mathrm{z}$ score $<-2)(\mathrm{P}=0.06)$. However, the median $\mathrm{z}$ score was the same in the two groups. No other significant differences in background data existed (table 1).

As the likelihood of diagnosing complications depends on the number of times the participants were seen, we defined a group with very good follow-up. This included 50 participants who had three or more visits within 10 days after inclusion.

Table 2 shows the characteristics of participants who developed pneumonia or were admitted to hospital. One participant was from the co-trimoxazole group, and six were from the placebo group; four had been vaccinated against measles, and three were unvaccinated.

\section{Laboratory confirmed measles}

Among the 84 cases, 67 (80\%) were serologically confirmed by either a fourfold rise in $\operatorname{IgG}$ titre $(\mathrm{n}=42)$ or positive $\operatorname{IgM}(\mathrm{n}=25)$. Seventeen $(20 \%)$ cases were not serologically confirmed. Three of these participants had no sample or insufficient blood for IgM analysis. Eight had only one sample before or within the first four days after the rash, and positive IgM could thus not be expected. One had very high IgG and negative IgM six days after the rash and only one blood sample. Three had very high IgG in both first and second sample, but the samples were not available for IgM testing. Two had samples from the day of rash or three days before and five to six weeks after; IgG titre did not increase four- 
Table 1 Characteristics of randomised participants. Values are numbers (percentages) unless stated otherwise

\begin{tabular}{|c|c|c|}
\hline Characteristic & $\begin{array}{l}\text { Co-trimoxazole } \\
\qquad(\mathrm{n}=46)\end{array}$ & Placebo $(\mathrm{n}=38)$ \\
\hline Male & $23(50)$ & $23(61)$ \\
\hline Median (range) age (years) & $4.4(0.64-24.8)$ & $5.9(0.49-18.8)$ \\
\hline Median (range) weight at inclusion $(\mathrm{kg})$ & $15.7(6.0-65.0)$ & $17.7(5.6-54.6)$ \\
\hline Median (range) weight for age $\mathrm{z}$ score & $\begin{array}{c}-1.64 \\
(-4.24 \text { to } 0.38)\end{array}$ & $\begin{array}{c}-1.39 \\
(-3.73 \text { to }-0.22)\end{array}$ \\
\hline Weight for age $z$ score $<-2$ & $17 / 41(41)$ & $7 / 34 \quad(21)$ \\
\hline $\begin{array}{l}\text { Median (range) No of days between first and } \\
\text { second visit }\end{array}$ & $4.0(1-36)$ & $4.0(1-22)$ \\
\hline Median (range) length of follow-up (days) & $33(9-66)$ & $36(7-66)$ \\
\hline Median (range) No of visits & $5(2-8)$ & $6(3-8)$ \\
\hline \multicolumn{3}{|l|}{ Day of inclusion in relation to rash: } \\
\hline Before rash & $2(5)$ & $8(22)$ \\
\hline Same day to two days after rash & $33(78)$ & $25(67)$ \\
\hline Three or more days after rash & $7(17)$ & $4(11)$ \\
\hline Median (range) interval between blood samples & $35(25-63)$ & $34(21-71)$ \\
\hline Exposure, index case (versus secondary case) & $26(57)$ & $20(53)$ \\
\hline Vaccinated against measles* & $27(59)$ & $22(58)$ \\
\hline
\end{tabular}

* Includes participants who claimed to have been vaccinated and those who had one or two vaccination dates registered.

fold, and IgM tests were negative; both participants were infected by a sibling and had typical symptoms of measles. Hence, we considered that all clinically diagnosed cases probably represented measles infection.
Treatment failure due to pneumonia or hospital admission One of 46 participants taking co-trimoxazole developed pneumonia compared with six of 38 children taking placebo (odds ratio 0.08 (95\% confidence interval 0 to 0.56$)$, controlled for age group) (table 3). When we excluded the three participants who got a wrong dose of the trial drug (fig 3 ), the odds ratio was 0.06 ( 0 to 0.48 ) controlled for age group and 0.06 ( 0 to 0.79 ) controlled for age group, weight for age $\mathrm{z}$ score, duration of disease, and measles vaccination status (data not shown). The number needed to treat was 7 (4 to 48 ). All three participants admitted to hospital had received placebo $(\mathrm{P}=0.09)$. Among 50 participants with very good follow-up, the odds ratio was 0.05 (0 to 0.47 ), adjusted for age group. Results were essentially the same among 67 participants with laboratory confirmed measles (table 3).

In a sensitivity analysis of all randomised participants, we assumed that the one participant lost to follow-up in the co-trimoxazole group developed pneumonia and the two lost to follow-up in the placebo group remained healthy (fig 3). The three participants who got the wrong dose of trial drug did not develop pneumonia. Thus, the odds ratio was 0.20 (0.03 to 1.00) controlled for age group.

\section{Other outcome measures}

Weight gains during the first month after inclusion were 15 (2 to 29) g per day in the placebo group and 32 (23 to 42) g per day in the co-trimoxazole group $(\mathrm{P}=0.04)$. When we divided the results

Table 2 Characteristics of participants with pneumonia

\begin{tabular}{|c|c|c|c|c|c|c|c|}
\hline & Girl & Boy & Boy & Boy & Boy & Boy & Boy \\
\hline Age (years) & 0.50 & 0.72 & 1.14 & 3.16 & 3.74 & 4.71 & 6.68 \\
\hline Randomisation group & Placebo & Placebo & Placebo & Placebo & Placebo & Co-trimoxazole & Placebo \\
\hline Weight for age $z$ score & -1.84 & -2.39 & -2.95 & -1.13 & -1.40 & -1.17 & -1.76 \\
\hline $\begin{array}{l}\text { Day of treatment failure (after } \\
\text { rash) }\end{array}$ & 5 & 8 & 2 & 3 & 9 & 3 & 8 \\
\hline Reason for treatment failure & $\begin{array}{l}\text { Admitted to hospital; } \\
\text { pneumonia }\end{array}$ & $\begin{array}{l}\text { Admitted to hospital; } \\
\text { pneumonia }\end{array}$ & $\begin{array}{l}\text { Admitted to hospital; } \\
\text { pneumonia }\end{array}$ & Pneumonia & Pneumonia & Pneumonia & Pneumonia \\
\hline Died of measles & No & No & No & No & No & No & No \\
\hline Intensity of exposure & Secondary & Index & Secondary & Index & Secondary & Secondary & Index \\
\hline Laboratory confirmed measles & Yes & Yes & Yes & No & Yes & Yes & Yes \\
\hline Physician's diagnosis of measles & Definitive & Definitive & Definitive & Definitive & Definitive & Definitive & Definitive \\
\hline $\begin{array}{l}\text { Vaccinated against measles } \\
\text { before disease }\end{array}$ & $\mathrm{No}^{*}$ & No & Yes & No & Yes & Yes & Yes \\
\hline $\begin{array}{l}\text { Interval between first and second } \\
\text { visit (days) }\end{array}$ & 4 & 3 & 1 & 2 & 3 & 2 & 4 \\
\hline Chest radiograph & Not done & Yes: pneumonia & Yes: pneumonia & Yes: pneumonia & Yes: pneumonia & Not done & Not done \\
\hline Blood culture & Not done & No: not possible & Yes: no growth & Yes: no growth & Not done & Not done & Not done \\
\hline
\end{tabular}

${ }^{*}$ Received a measles vaccination at age 6 months (day before entry in this study) in an early two dose trial11 and developed rash four days later; infected by her sister.

Table 3 Intention to treat analyses of outcome measures. Values are numbers (percentages) unless stated otherwise Co-trimoxazole $(\mathrm{n}=46) \quad$ Placebo $(\mathrm{n}=38) \quad$ Odds ratiot $(95 \% \mathrm{CI}) \quad$ Adjusted odds ratiof $(95 \% \mathrm{Cl})$

Adjusted odds ratioł $(95 \% \mathrm{Cl})$ : laboratory confirmed cases

\begin{tabular}{|c|c|c|c|c|c|}
\hline \multirow{2}{*}{\multicolumn{6}{|c|}{ Main outcome measures }} \\
\hline & & & & & \\
\hline Pneumonia after inclusion & $1(2)$ & $6(16)$ & 0.08 (0 to 0.56$) \S$ & $0.14(0.01$ to 1.50$) \S$ & 0.11 (0 to 1.22$) \S$ \\
\hline $\begin{array}{l}\text { Admitted to hospital with measles after } \\
\text { inclusion }\end{array}$ & $0(0)$ & $3(8)$ & 0 (0 to 1.03$)$ & - & - \\
\hline \multicolumn{6}{|l|}{ Other outcome measures } \\
\hline Diarrhoea after inclusion & $3(7)$ & $5(13)$ & 0.27 (0.04 to 1.39)§ & 0.17 (0.01 to 1.55)§ & 0.10 (0 to 1.04$) \S$ \\
\hline Severe fever after inclusion & $6(13)$ & $11(29)$ & 0.32 (0.10 to 1.07$)$ & 0.36 (0.09 to 1.43) & 0.34 (0.08 to 1.53$)$ \\
\hline Oral thrush after inclusion & $0(0)$ & $3(8)$ & 0 (0 to 1.03$)$ & - & \\
\hline Stomatitis after inclusion & $4(9)$ & $7(18)$ & 0.37 (0.09 to 1.50) & $0.43(0.08$ to 2.26$)$ & 0.35 (0.06 to 2.12) \\
\hline Conjunctivitis after inclusion & $12(26)$ & $17(45)$ & $0.36(0.14 \text { to } 0.96)^{*}$ & 0.31 (0.10 to 1.03$)$ & $0.25(0.06 \text { to } 0.96)^{\star}$ \\
\hline Otitis media after inclusion & $1(2)$ & $2(5)$ & 0.38 (0.02 to 4.42$) \S$ & $0.72(0.05$ to 10.6$) \S$ & 0.44 (0.01 to 6.93$) \S$ \\
\hline
\end{tabular}

${ }^{*} \mathrm{P}<0.05$.

tControlled for age group.

$\ddagger$ Adjusted for age group, weight for age z score at inclusion, time since rash, and measles vaccination status.

$\S$ Profile-likelihood confidence interval. 
Table 4 Weight change per age group between co-trimoxazole and placebo groups

\begin{tabular}{lccc}
\multirow{2}{*}{ Age group } & \multicolumn{3}{c}{ Weight change (g/day), adjusted } \\
\cline { 2 - 4 } & Co-trimoxazole & Placebo & $\begin{array}{c}\text { Co-trimoxazole }- \text { placebo } \\
(\mathbf{9 5 \%} \text { Cl) }\end{array}$ \\
\hline 2-11 months $(n=9)$ & 9 & 26 & $-18(-56$ to 21$)$ \\
\hline $1-2$ years $(n=9)$ & 28 & 8 & $20(-2$ to 42$)$ \\
\hline $3-4$ years $(n=17)$ & 28 & 0 & $27(-11$ to 67$)$ \\
\hline $5-17$ years $(n=40)$ & 65 & 27 & $39(10 \text { to } 68)^{*}$ \\
\hline All ages $(n=75)$ & 32 & 15 & $17(1 \text { to } 33)^{\star}$ \\
\hline
\end{tabular}

${ }^{*} \mathrm{P}<0.05$.

†Adjusted for age group, weight for age at inclusion, time since rash, and measles vaccination status.

into age groups, a non-significant weight loss occurred in the 2-11 months age group in the co-trimoxazole group compared with the placebo group; in the other age groups a weight gain occurred, which was significant in the 5-17 years age group (table 4). When we excluded participants who got the wrong dose of trial drug, the results were essentially the same among 50 participants with very good follow-up and the 67 participants with laboratory confirmed measles (data not shown).

We found significantly less conjunctivitis and a nonsignificant tendency to less diarrhoea, oral thrush $(\mathrm{P}=0.09)$, severe fever, and stomatitis among recipients of co-trimoxazole. Otitis media did not differ between the groups (table 3). We grouped participants according to number of complications. Forty four participants had no complications, 22 had one complication, eight had two, five had three, one had four, two had five, and two had seven complications. Among 40 participants with any complication, 21 had received placebo and 19 had received co-trimoxazole (odds ratio 0.47 ( 0.18 to 1.18 ), adjusted for age group). Eight participants in the placebo group had three or more complications, as did two in the co-trimoxazole group $(\mathrm{P}=0.04)$. Among participants with complications, $19(48 \%)$ were vaccinated (eight had a history of vaccination, nine had a measles vaccination date, and two had been vaccinated twice). Assuming that those without a vaccination date had been vaccinated at 9 months of age, the median time between the first vaccination and the first measles complication was 5.5 (interquartile range 2.2-7.3) years. Among 44 participants without complications, $25(57 \%)$ were vaccinated (11 had a history of vaccination, $10 \mathrm{had}$ a vaccination date, and four were vaccinated twice). Vaccinated participants thus had a marginally lower risk of complications than unvaccinated ones (relative risk 0.82 (0.52 to 1.29)). The effect of co-trimoxazole was the same among vaccinated and unvaccinated participants (test for interaction, $\mathrm{P}=0.45$ ).

\section{Discussion}

Participants with measles who received co-trimoxazole had less pneumonia, less conjunctivitis, and more weight gain than those who received placebo, indicating a beneficial effect of prophylactic antibiotics in the management of measles in low income countries. The number needed to treat was 7 , so for every seven patients with measles treated with prophylactic antibiotics one case of pneumonia was prevented.

\section{Nutritional status}

On inclusion, by chance, a larger proportion of participants in the co-trimoxazole group were malnourished (weight for age $\mathrm{z}$ score $<-2$ ). Some people consider malnutrition, as well as size of infecting dose, ${ }^{15}$ to be a risk factor for severe measles and death from measles, and the co-trimoxazole group could thus be expected to develop more complications than the placebo group, whereas the opposite was true. Our data show a benefit from receiving prophylactic antibiotics, an effect that might have been underestimated as a result of the uneven proportion of malnourished patients in the two groups.

Overall, a significantly larger weight gain occurred in the co-trimoxazole group. Non-significant weight loss occurred among infants who received co-trimoxazole compared with placebo, which is a matter for concern.

Diagnosis of measlesAlthough $20 \%$ of the cases of measles were not confirmed serologically, all patients with measles had typical symptoms. Diagnosis of measles is increasingly difficult because of efforts to eradicate the disease ${ }^{16}$ but the likelihood of diagnosing measles is higher in an epidemic situation such as the one described here than for sporadic cases. ${ }^{17}$ Although the IgM ELISA test can give false positives ${ }^{18}$ the combination of the clinical picture, exposure to measles, and the serological tests gives a high likelihood of the diagnosis of measles being correct. Furthermore, the results were essentially the same in analyses of all 84 randomised participants, the 81 participants who received the right dose of trial drug, the 67 participants with laboratory confirmed measles (table 3 ), and the 50 participants with very good follow-up.

\section{Strengths and weaknesses}

The small sample size of 84 patients with measles is a serious limitation to this study. Even so, all but one case of pneumonia and all the hospital admissions occurred in the placebo group. This, combined with data showing significantly less conjunctivitis and a larger weight gain in the co-trimoxazole group, indicates a beneficial effect of prophylactic antibiotics. As this was a randomised, double blind, placebo controlled trial, we do not have any reason to believe that restricting the data analysis to the year 1998 could have biased our results. Sensitivity analysis including all 87 randomised participants (fig 3) shows that the effect of prophylactic antibiotics on pneumonia remained strong, although diminished. Thus, when we estimated the worst case scenario with the available data, $80 \%$ less pneumonia still occurred in the co-trimoxazole group.

The risk of developing complications was 18\% lower among vaccinated than unvaccinated participants. This was to be expected; however, once a patient had acquired measles, the effect of co-trimoxazole was the same among vaccinated and unvaccinated participants.

Several questions remain because of the limited study size. The study does not provide mortality results, as no participant died. If prophylactic antibiotics reduce the occurrence of measles associated pneumonia by about $90 \%$, a reduction in mortality from measles would be expected, as pneumonia is the main killer in complicated measles in low income countries. ${ }^{19}$ In the Senegalese study, case fatality rates fell twofold and respiratory symptoms threefold with the introduction of prophylactic antibiotics. $^{8}$

\section{Antibiotic resistance}

The development of antibiotic resistance is enhanced by uncritical use of antibiotics. Widespread resistance to co-trimoxazole exists, ${ }^{20}$ but no clear association exists between antimicrobial resistance and clinical outcome of pneumonia. ${ }^{21}$ Bacterial superinfection in viral pneumonia is very common, not least in measles, ${ }^{22}$ and if co-trimoxazole could prevent a large proportion of bacterial pneumonia and pneumonia related deaths it is probable that the strategy would be highly cost effective, as co-trimoxazole is not an expensive drug. Despite the increasing resistance, co-trimoxazole is still recommended by 


\section{Research}

WHO as a first line drug for community acquired pneumonia. ${ }^{23}$ Whether prophylactic treatment with co-trimoxazole will add to the development of resistance is questionable, as the drug is already widely used. Antimicrobial resistance to amoxicillin is less common; amoxicillin is the other drug recommended first line for community acquired pneumonia by WHO and may be an alternative to co-trimoxazole.

\section{Conclusions}

Even though a Cochrane review concluded that antibiotics should be given only if clinical signs of pneumonia or other evidence of sepsis are present, ${ }^{724}$ we believe that the evidence, including that from this study, favours the use of prophylactic antibiotics in measles in low income countries. Prophylactic antibiotics should be used in patients with measles, disregarding vaccination status, in settings with a high risk of complications when the diagnosis of measles is quite certain, such as during epidemics. The international community needs to decide whether more placebo controlled randomised studies of prophylactic antibiotics in measles are needed given our present knowledge. Such trials might examine different drug regimens and their impact in different age groups, patterns of antibiotic resistance, and mortality from measles to get an idea if the case fatality rate declines.

We thank Mario Monteiro, Laboratório Nacional de Saúde Pública, Guinea-Bissau, and Elishia Roberts and Tisbeh Faye-Joof, Medical Research Council Laboratories, Gambia, for collection and analyses of blood samples; Kim Mulholland and Martin Weber, Medical Research Council Laboratories, Gambia, for clinical training in diagnosing pneumonia; Anita Sandström for producing the randomisation lists; and Palle ValentinerBranth for computerising the randomisation lists.

Contributions: M-LG, PA, and HCW designed the study. M-LG, CB, CLM, IML, and PA implemented and conducted the study. M-LG and JN analysed the data. M-LG wrote the first draft, and all authors contributed to the final version of the paper. M-LG is the guarantor.

Funding: None.

Competing interests: None declared.

Ethical approval: Ministry of Health, Guinea-Bissau; Danish Central Ethical Committee; and Scientific Co-ordination Committee, Medical Research Council, Gambia.

\section{What is already known on this topic}

Studies of prophylactic antibiotics in measles have been inconclusive when evaluated by current standards

An observational study from Senegal found a twofold reduction in measles case fatality rate and a threefold reduction in respiratory symptoms with prophylactic antibiotics

\section{What this study adds}

Prophylactic antibiotics in measles infection prevented pneumonia, conjunctivitis, and possibly other complications and improved weight gain in the month after measles infection in a low income setting
1 Progress in reducing global measles deaths: 1999-2004. Wkly Epidemiol Rec 2006; $10: 90-4$

2 Global measles mortality reduction and regional elimination, 2000-2001: part I. Wkly Epidemiol Rec 2002;77:50-5.

3 Loening WE, Coovadia HM. Age-specific occurrence rates of measles in urban, peri-urban, and rural environments: implications for time of vaccination. Lancet 1983;ii:324-6.

4 Van Den HS, Smit C, Van Steenbergen JE, De Melker HE. Hospitalizations during a measles epidemic in the Netherlands, 1999 to 2000. Pediatr Infect Dis J 2002:21:1146-50.

5 Aaby P, Bukh J, Leerhoy J, Lisse IM, Mordhorst CH, Pedersen IR. Vaccinated children get milder measles infection: a community study from Guinea-Bissau. J Infect Dis 1986:154:858-63.

6 Chalmers I. Why we need to know whether prophylactic antibiotics can reduce measles- related morbidity. Pediatrics 2002;109:312-5.

7 Shann F, D'Souza RM, D'Souza R. Antibiotics for preventing pneumonia in children with measles. Cochrane Database Syst Rev 2006; (3): CD001477.

8 Samb B, Simondon F, Aaby P, Whittle HC, Seck AMC. Prophylactic use of antibiotics and reduced case fatality in measles infection. Pediatr Infect Dis J 1995;14:695-6.

World Health Organization. Clinical research on treatment of measles: report of a meeting. Geneva: WHO, 1995. (WHO/CDR/95.15)

10 Aaby P. Bandim. An unplanned longitudinal study. In: Das Gupta M, Aaby P, Pison G, Garenne M, eds. Prospective community studies in developing countries. Oxford: Clarendon Press, 1997:276-96.

11 Garly M-L, Martins CL, Balé C, da Costa F, Dias F, Whittle HC, et al. Early two-dose measles vaccination schedule in Guinea-Bissau: good protection and coverage in infancy. Int J Epidemiol 1999;28:347-52

12 Samb B, Aaby P, Whittle HC, Seck AM, Simondon F. Protective efficacy of high-titre measles vaccines administered from the age of five months: a community study in rural Senegal. Trans R Soc Trop Med Hyg 1993;87:697-701.

13 Whittle HC, Mann G, Eccles M. Effects of dose and strain of vaccine on success of measles vaccination of infants aged 4-5 months. Lancet 1988;i:963-6.

14 Helfand RF, Heath JL, Anderson LJ, Maes EF, Guris D, Bellini WJ. Diagnosis of measles with an IgM capture EIA: the optimal timing of specimen collection after rash onset. $J$ Infect Dis 1997;175:195-9.

15 Morley D, Aaby P. Managing measles: size of infecting dose may be important. BMJ 1997;314:1692.

16 Hutchins SS, Papania MJ, Amler R, Maes EF, Grabowsky M, Bromberg K, et al. Evaluation of the measles clinical case definition.J Infect Dis 2004;189(suppl 1):153-9.

17 Nur YA, Groen J, Yusuf MA, Osterhaus AD. IgM antibodies in hospitalized children with febrile illness during an inter-epidemic period of measles, in Somalia. J Clin Virol 1999;12:21-5.

18 Dietz V, Rota J, Izurieta H, Carrasco P, Bellini W. The laboratory confirmation of suspected measles cases in settings of low measles transmission: conclusions from the experience in the Americas. Bull World Health Organ 2004;82:852-7.

19 Perry RT, Halsey NA. The clinical significance of measles: a review. J Infect Dis 2004;189(suppl 1):4-16

20 Qazi SA. Antibiotic strategies for developing countries: experience with acute respiratory tract infections in Pakistan. Clin Infect Dis 1999;28:214-8.

21 Klugman KP. The clinical relevance of in-vitro resistance to penicillin, ampicillin, amoxycillin and alternative agents, for the treatment of community-acquired pneumonia caused by Streptococcus pneumoniae, Haemophilus influenzae and Moraxella catarrhalis. J Antimicrob Chemother 1996;38(suppl A):133-40.

22 Quiambao BP, Gatchalian SR, Halonen P, Lucero M, Sombrero L, Paladin FJ, et al. Coinfection is common in measles-associated pneumonia. Pediatr Infect Dis $J$ 1998:17:89-93.

23 World Health Organization. Integrated management of childhood illness. 2004. www.who.int/child-adolescent-health/integr.htm (accessed 9 Oct 2006].

24 Shann F. Meta-analysis of trials of prophylactic antibiotics for children with measles inadequate evidence. BMJ 1997;314:334-7.

(Accepted 28 September 2006)

doi 10.1136/bmj.38989.684178.AE

Projecto de Saúde de Bandim, Apartado 861, Bissau, Guinea-Bissau

May-Lill Garly postdoctoral researcher

Carlitos Balé project physician

Cesário Lourenco Martins director

Jens Nielsen statistician

Ida M Lisse researcher

Peter Aaby research professor

Medical Research Council Laboratories, Fajara, Gambia

Hilton C Whittle visiting professor

Correspondence to: M-L Garly, Bandim Health Project, Division of Epidemiology,

Statens Serum Institut, Artillerivej 5, 2300 Copenhagen S, Denmark mlg@ssi.dk 